\title{
Expert Discussion: HER2-Positive Breast Cancer
}

\author{
Theodoros Foukakis ${ }^{\mathrm{a}, \mathrm{b}} \quad$ Alexios Matikas $^{\mathrm{a}, \mathrm{b}}$ Olga Oikonomidou $^{\mathrm{c}}$ \\ Eleni Andreopoulou d Dimitris Mavroudis ${ }^{\mathrm{e}}$ \\ ${ }^{a}$ Department of Oncology-Pathology, Karolinska Institute, Stockholm, Sweden; ${ }^{b}$ Breast Center, Theme Cancer, \\ Karolinska University Hospital, Stockholm, Sweden; ${ }^{C}$ Cancer Research UK Edinburgh Centre - Institute of \\ Genetics and Cancer, Edinburgh Cancer Centre, Western General Hospital, University of Edinburgh, \\ Edinburgh, UK; ${ }^{d}$ Breast Cancer Center Clinical Research, Weill Cornell Medicine/New York Presbyterian Hospital, \\ New York, NY, USA; ${ }^{e}$ Department of Medical Oncology, University Hospital of Heraklion and Medical School, \\ University of Crete, Heraklion, Greece
}

\section{Foukakis: What is the role of genomic signatures and mutations in the treatment of early HER2-positive (HER2+) breast cancer (BC)?}

Matikas: Despite the strong prognostic and predictive value of HER2 positivity as assessed in the clinic (protein overexpression and/or gene amplification), it is now obvious that HER2 $+\mathrm{BC}$ is molecularly heterogeneous. The HER2-enriched intrinsic subtype only partially overlaps with clinical HER2+ BC and is a powerful predictor of benefit from HER2-blockade, especially when combined with the level of ERBB2 amplification. At the same time, common mutations such as at PIK3CA have been found to confer resistance to treatment with trastuzumab or trastuzumab emtansine. Even the intratumoral heterogeneity of HER2 expression has been associated with resistance to chemotherapy-free neoadjuvant therapy with trastuzumab emtansine. In the near future, I am anticipating that predictive information at the genomic (mutations) and transcriptomic (intrinsic subtype, gene signatures) levels will be combined with information from standard pathology (immune infiltrate, HER2 heterogeneity) to construct predictive models which will select patients exquisitely sensitive to HER2 blockade alone, thus sparing them from unnecessary chemotherapy. Such combinatory approaches have already been shown to be feasible and clinically relevant, for example HER2DX by Prat et al. [1].
Oikonomidou: Starting from the neoadjuvant setting, there have been significant efforts to identify genomic predictors of pathologic complete response (pCR) that will guide treatment de-escalation. Loibl et al. [2] combined individual patient data from 5 clinical trials evaluating PIK3CA mutations and associations with pCR, disease-free survival (DFS), and overall survival (OS). They confirmed PIK3CA mutations to be associated with a reduced rate of pCR, mainly in the hormone receptor $(\mathrm{HR})+$ group but no conclusions were drawn regarding survival. On the adjuvant setting now and in ExteNET trial, the benefit of neratinib over placebo was greater in PIK3CA-altered tumors than PIK3CA wild-type tumors but the interaction test was not significant. We do know that $\mathrm{HR}+/ \mathrm{HER} 2+$ behave differently to HR-/HER2 with lower pCR rates and lower benefit to dual HER2 blockade. It has been suggested that the HR+/HER2+ tumors with high ESR1 levels get less benefit from adjuvant trastuzumab. On the advanced setting, phase III trial SOFIA investigated the role of margetuximab plus chemotherapy in heavily pretreated HER $2+$ metastatic $\mathrm{BC}$. The exploratory analysis by CD16A genotype showed that the benefit was enhanced in patients with low-affinity CD16A genotypes containing a $158 \mathrm{~F}$ allele. A neoadjuvant trial is being planned to study margetuximab in patients with HER2+ BC who carry the CD16A-158F allele. Tumor infiltrating lymphocytes (TILs) may have a predictive role in HER2+ BC. The GBCG showed that in- 
creased TIL concentration predicted response to NACT with $48 \%$ of the group with high TILs achieving pCR and this was also correlated with survival benefit. Last but not least, the clinical subtype plays a role and we do know that the HER2 group represents multiple biologic intrinsic subtypes that most certainly have clinical implications. The pCR rates were higher (more than double) in the HER2-enriched subtype than either luminal subtype in CALGB 40601, NOAH, and NeoALLTO. In PAMELA trial patients with HER2-enriched tumors had $41 \%$ pCR after receiving lapatinib/trastuzumab $\pm \mathrm{ET}$ (if $\mathrm{HR}+$ ) compared to $10 \%$ of patients with luminal or basal-like subtypes.

Andreopoulou: Although of unquestionable value, immunohistochemistry is inadequate to capture the complexity of HER2+ BC and the heterogeneity within the subset. Accurate risk stratification is mandatory to correctly identify optimal candidates for escalated or deescalated strategies. Numerous promising biomarkers, such as molecular subtyping, TILs, and on-treatment markers have been identified and need to be integrated in the design of future trials as selection or stratification factors. Analysis of the genomic landscape in this subset identified molecular features that differentiate cases that achieved a pCR from those that had residual disease. A set of recurrent somatic events in HER2 + BC may aid in the identification of patients, at the time of diagnosis, who will not have a pCR after treatment with chemotherapy and trastuzumab. ERBB2 and GRB7 were the genes most commonly observed in fusion events, and genomic copy number analysis of the ERBB2 locus indicated that cases with either no observable or low-level ERBB2 amplification were less likely to achieve a pCR. Notably in pCR cases, tumors consistently expressed immune signatures that may contribute to therapeutic response. In particular these cases had evidence of an activated immune response, including $\mathrm{T}$-cell, $\mathrm{B}$-cell, and inflammatory signatures. In contrast, cases with residual disease displayed evidence of greater heterogeneity as no RNA signatures were enriched among this group. HER2enriched cases achieved significantly higher rates of pCR compared to those classified as luminal A, luminal B, basal-like, or normal-like. PAM50 molecular subtyping has also been addressed in this context. TILs have been associated with increased pCR rates and improved outcomes. For de-escalated treatments, molecular biology is a key factor. Molecular heterogeneity of HER2+ BC, beyond the hormone receptor status, is a major determinant of sensitivity to anti-HER2 treatments. Studies demonstrated that Ki67 inhibition after short-term letrozole may allow to identify patients with meaningful pCR without chemotherapy.

Expert Discussion: HER2-Positive Breast Cancer
Mavroudis: Next-generation sequencing-based assays indicate that HER2 somatic mutations are present in approximately $2-5 \%$ of primary BCs. The vast majority of HER2 somatic mutations have been reported in HER2 amplification-negative patients.

Neratinib, a next-generation, irreversible pan-HER tyrosine kinase inhibitor, inhibits EGFR, HER2, and HER4, preventing autophosphorylation and activation of oncogenic intracellular signaling pathways. The ExteNET study showed that neratinib significantly improved 5 -year invasive DFS (iDFS) in women who completed trastuzumab-based adjuvant therapy for early BC. Neratinib is used in the US for extended adjuvant treatment of adult patients with early-stage HER $2+$ BC following 1 year of trastuzumab. In Europe, neratinib is used in the extended adjuvant treatment of adult patients with earlystage hormone receptor-positive HER2 $+\mathrm{BC}$ who are less than 1 year from the completion of prior adjuvant trastuzumab-based therapy. More recently, based on the phase II NALA study neratinib received an expanded indication for use in combination with capecitabine in patients with metastatic HER2+ BC who have already failed 2 or more previous HER2-targeted regimens for metastatic disease.

In the SUMMIT study neratinib was used to treat HER2 and HER3 mutant cancers of different histology. Neratinib was active (32\% response rate among 25 patients) in patients with HER2-mutated BC with missense mutations in the extracellular and kinase domains and insertions in the kinase domain [3]. Based on these early results neratinib is a promising agent for targeting HER2 mutated BC.

\section{Foukakis: What are the most promising approaches for treatment de-escalation in early HER2+ BC?}

Matikas: Ongoing de-escalation efforts for treatment of early HER2+ BC are multifaceted. At the neoadjuvant setting, carboplatin-based and anthracycline-free regimens have been shown to produce similar pCR rates as sequential taxanes and anthracyclines in TRAIN-2. Long-term outcomes were not compromised and fewer heart-related adverse events were noted with anthracycline-free adjuvant therapy in BCIRG-006. In addition, KRISTINE and PREDIX HER2 demonstrated the feasibility of chemotherapy-free neoadjuvant therapy, based on the antibody-drug conjugate (ADC) trastuzumab emtansine. Optimizing the use of ADCs as detailed in question 1, expanding their use to the adjuvant setting for low-risk HER2+ BC (ATEMPT) and evaluating novel ADCs such as trastuzumab deruxtecan represent major strides towards de-escalation. Furthermore, the conflicting results from PHARE and PERSEPHONE imply that 
some patients may benefit from shorter adjuvant trastuzumab duration. Highly anticipated data from the upcoming Oxford group meta-analysis will hopefully shed light on this issue. Whether any adjuvant therapy per se is needed for patients attaining a pCR is unclear and represents a highly promising research avenue for future deescalation.

Oikonomidou: An important study is the APT trial that recruited low-risk HER $2+$ BC patients and assessed whether these could be treated with paclitaxel and trastuzumab alone. Investigators reported a 3-year iDFS of $98.7 \%$. At 7 years, DFS was $93.3 \%$ and OS was $95 \%$. ATEMPT trial sought to determine whether T-DM1 would be less toxic than paclitaxel plus trastuzumab at stage I HER2+ BC patients and to evaluate the DFS in patients receiving T-DM1. In the $\sim 400$ patients that received T-DM1, the 3 -year DFS was $97.7 \%$ with only 2 distant recurrences but $17 \%$ of patients discontinued treatment due to toxicity compared with $6 \%$ of patients who received paclitaxel and trastuzumab combination. T-DM1 might be a good alternative but toxicities and cost should be taken in account. Another effort of deescalation was Persephone, an open-label, randomized phase III non-inferiority trial. More than 4,000 women received either 6 or 12 months of adjuvant trastuzumab, with 4 -year DFS of $89.4 \%$ versus $89.8 \%$, meeting noninferiority endpoints of the study. There were also less events of cardiotoxicity and fewer serious adverse events (5\% less). The PHARE trial tested the same hypothesis to Persephone in $\sim 3,400$ patients with a different result: the hazard ratio for DFS at 7.5 years was 1.28 , worse than the noninferiority cut off. At this time, for patients with cardiac disease or poor treatment tolerance having shorter duration of trastuzumab seems to provide most of the benefit.

Andreopoulou: De-escalated anti-HER2 strategies include:

\section{Shorter Anti-HER2 Therapy}

Adjuvant 1-year trastuzumab remains the standard. A shorter trastuzumab treatment for patients at low risk of relapse and/or high risk of cardiac toxicity has been suggested. Subsequently to FinHER Trial that supported significant improvement in DFS and no cardiac toxicity with only 9 weeks of adjuvant Herceptin a number of randomized trials have investigated the noninferiority of a shorter duration of trastuzumab versus 1 year but it was only the PERSEPHONE trial that proved the non-inferiority of shorter trastuzumab. In the ShortHER study patients with low TILs appeared to benefit from 1-year trastuzum$\mathrm{ab}$, whereas in case of high TILs prognosis was extremely good with both treatments.
De-Escalating Chemotherapy - Anthracycline-Free Chemotherapy

The trastuzumab-containing anthracycline-free arm of the BCIRG006 trial was reported superior to anthracycline-based chemotherapy alone and showed a safer profile as compared to trastuzumab added to anthracycline and taxane. Considering the favorable safety profile, the ATP regimen of weekly paclitaxel for 12 weeks and concomitant trastuzumab for 1 year has entered clinical use for low-risk patients, although some concerns still exist on the indication for patients with tumors of $2-3 \mathrm{~cm}$ and in case of negative estrogen receptor. A currently ongoing trial compares 1-year T-DM1 with the APT regimen for stage I patients.

De-Escalating Chemotherapy - Chemotherapy-Free Regimens

In the NeoSphere trial the chemotherapy-free arm with trastuzumab + pertuzumab led to a significant pCR rate of $16.8 \%$ and mostly interesting in the HR-positive group a pCR of $27.3 \%$ prompting the investigation of chemotherapy-free neoadjuvant regimens, most frequently exploiting the efficacy of dual blockade. A number of studies specifically focused on HER2+/HR+, a subgroup recognized as less sensitive to chemotherapy, supported by the rationale to potentially exploit the synergism between anti-HER 2 and endocrine therapy. In fact, the evaluation of a combination of neoadjuvant trastuzum$\mathrm{ab} /$ pertuzumab/fulvestrant/palbociclib for preoperative treatment of HER $2+/ \mathrm{HR}+$ resulted in pCR of $27 \%$.

Mavroudis: The standard of care for both adjuvant and neoadjuvant therapy of early HER $2+B C$ is the sequential administration of an anthracycline regimen followed by a taxane in combination with anti-HER2 agent(s). The following represent de-escalation therapeutic options.

\section{In the Adjuvant Setting}

For patients with stage II and III disease, the use of the non-anthracycline regimen of docetaxel plus carboplatin plus trastuzumab and pertuzumab (TCHP) extrapolating from the BCIRG-006 trial, which showed similar efficacy but reduced toxicity for $\mathrm{TCH}$ versus ACTH [4].

For patients with node-negative, HER2-positive tumors $<2 \mathrm{~cm}$ adjuvant treatment with paclitaxel $(80 \mathrm{mg} /$ $\mathrm{m}^{2}$ ) weekly for 12 weeks plus trastuzumab (with or without pertuzumab) for 1 year based on the study by Tolaney et al. [4], which reported 7-year DFS of $93 \%$ and OS of 95\%.

\section{In the Neoadjuvant Setting}

The use of docetaxel, carboplatin, trastuzumab, and pertuzumab (TCHP regimen), which avoids the use of anthracyclines based on the TRYPHAENA trial [5]. The 
reported pCR rate for TCHP was 64 versus $55 \%$ for FECTHP with similar or reduced toxicity.

For patients with comorbid conditions or who wish to avoid chemotherapy the neo-adjuvant administration of dual HER2-targeted therapy based on the NeoSphere trial [6], which showed a pCR rate 6\% for HR-positive and $27 \%$ for HR-negative tumors. However this pCR rate was inferior to the combination of chemotherapy and HER2targeted therapy.

\section{Foukakis: With several novel agents targeting HER2 recently approved or awaiting approval during 2021, how will you choose treatment in metastatic HER2+ BC from third line and beyond?}

Matikas: This is an exciting time for the treatment of metastatic HER2+ BC. Following progression on trastuzumab/pertuzumab-based therapy and trastuzumab emtansine, we have now access to no fewer than 5 HER2targeted therapies: lapatinib, neratinib, tucatinib, margetuximab, and trastuzumab deruxtecan, while continuing with chemotherapy/trastuzumab combinations remains a valid option. Another emerging option for hormone receptor-positive HER2+ BC is CDK4/6 inhibition combined with fulvestrant and trastuzumab (monarcHER), while the role of checkpoint inhibitors remains uncertain (KATE2). Few direct comparisons exist. In accordance with current ESMO guidelines, tucatinib and trastuzumab deruxtecan are preferred options - the latter pending the publication of the randomized DESTINY-Breast 02 trial. Tucatinib has demonstrated an overall survival benefit when added to capecitabine/trastuzumab, tolerable toxicity profile, and meaningful activity against brain metastases, in a clinically relevant population that had received a median of 4 treatment lines, including the current standard of care. Trastuzumab deruxtecan has also shown potentially game-changing results in a single-arm trial of heavily pretreated patients. Both agents are being evaluated at earlier lines, even in combination with each other (NCT04539938). In the end, regional approvals, costs, and toxicity profiles will be deciding factors in managing patients at the third line and beyond.

Oikonomidou: Very exciting time for novel therapies to treat HER2+ metastatic BC. The current standard of care is dual blockade with trastuzumab + pertuzumab as first-line followed by TDM-1 as second-line. Several choices are available in third-line or more. These include trastuzumab-deruxtecan (T-Dxd), tucatinib, and neratinib (not really impressed by the Mergetuximab data). Impressive data from Destiny-01 and T-Dxd in heavily pretreated patients with median of 6 prior lines. ORR was about $61 \%$ and duration of response was 14.8 months, which is quite long when considering how heavily pretreated these patients were. PFS was 16.4 months and interestingly in those patients who had central nervous system (CNS) metastases that were stable and previously treated, the PFS was 18 months. Extra vigilance is required when it comes to the risk of ILD. Tucatinib is showing very exciting data from HER2-CLIMB trial with OS (18 vs. 12 months) and PFS benefit and substantial benefit in patients with brain metastases (14 vs. $\sim 6$ months in those with stable brain disease to 9.5 vs. 4.1 months in those with active brain metastases). The absolute PFS benefit is modest at 2.2 months. With regards to toxicity and mainly grade $3 / 4$ diarrhea, there was only about a $4 \%$ difference between the tucatinib arm and placebo. Now, neratinib and capecitabine combination has been FDA approved based on NALA trial data $(2 \mathrm{~L}+)$. Results showed an improved PFS by 2 months in the neratinib arm despite the fact that a lower dose of capecitabine was used and that interestingly the cumulative incidence for intervention for CNS metastases was lower in the neratinib arm. Grade 3/4 diarrhea was pretty high with $24 \%$ having grade $3 / 4$. The OS was not different, though, between the 2 arms. Based on the above in the third-line setting, I would choose between T-Dxd or tucatinib/capecitabine/trastuzumab based on whether or not a patient has brain metastases and whether these are stable or active. Patient's preference will also influence my decision. I will consider the neratinib/ capecitabine combination on the third line in patients with no CNS disease and if very keen on receiving oral therapy.

Andreopoulou: Although several options are available in the third-line setting there is no standard approach. Many of these treatments have not been directly compared with each other. Third-line options include lapatinib/capecitabine, trastuzumab/capecitabine, trastuzumab/lapatinib, trastuzumab/chemotherapy, and also T-DM1 and pertuzumab (for those who did not received it before). Continuing trastuzumab into further lines of therapy with alternative chemotherapy and biologic treatment backbones remains of course a standard practice while there is ongoing clinical development of several drugs in the third-line setting of HER2+ BC; some already have shown activity in phase III. New appealing options available include Tucatinib in the triple combination with capecitabine and trastuzumab based on the results of the HER2CLIMB trial. The newer ADC that was approved this year, trastuzumab deruxtecan, showed impressive effectiveness in heavily pretreated patients with HER2+ BC. As new drugs in development are continuously expanding the available options we will ultimately be called to select and sequence in a personalized care 
mode. A novel anti-HER2 antibody called margetuximab is characterized by enhanced binding to the activating low-affinity Fc receptor CD16A on immune effector cells potentially mounting a strong immune response against the cancer.

Mavroudis: This is a real puzzle because data are lacking for the optimal use of all the emerging options with the new treatments. The following drugs have recently gained approval for use in this setting.

Trastuzumab deruxtecan is an ADC composed of an anti-HER2 antibody, a cleavable tetrapeptide-based linker, and a cytotoxic topoisomerase I inhibitor. It is approved for use in patients with metastatic HER $2+\mathrm{BC}$ who have progressed after 2 or more prior anti-HER2-based regimens typically including a taxane plus trastuzumab/ pertuzumab and T-DM1 based on the multicenter phase 2 Destiny Breast001 study, which included 184 heavily pretreated patients (median prior lines of therapy 6) and was presented at SABCS 2019. In this patient population the reported response rate was $60.9 \%$ with a median duration of response of 14.8 months and median PFS 16.4 months. The most worrisome adverse effect was interstitial lung disease/pneumonitis affecting $13.6 \%$ of patients. Update data from this study at SABCS 2020 showed a median PFS 19.4 months and a median OS 24.6 months. Patients should be closely monitored for the development of interstitial lung disease, which was fatal in $2.7 \%$ of patients.

Tucatinib is an oral tyrosine kinase inhibitor that is selective for the kinase domain of HER2, with minimal inhibition of epidermal growth factor receptor. It is approved for use in combination with trastuzumab and capecitabine for treatment of patients with metastatic HER2+ BC who have received one or more prior antiHER2-based regimens based on the randomized HER2 CLIMB phase II study, which included 610 patients (median prior lines of therapy 4 ) and was presented at SABCS 2019. The addition of tucatinib to capecitabine plus trastuzumab resulted in an objective response rate of 41 versus $23 \%$, a median PFS of 7.8 versus 5.6 months, and median OS 21.9 versus 17.4 months for tucatinib versus placebo arm. Even among 291 patients with untreated or treated brain metastases the median PFS was 7.6 versus 5.4 in favor of tucatinib. Diarrhea, palmar-plantar erythrodysesthesia, and nausea were the most common side effects for the tucatinib arm.

Margetuximab is a new anti-HER2-receptor monoclonal antibody with higher affinity than trastuzumab for Fc receptor CD16A that is FDA approved, in combination with chemotherapy, for treatment of metastatic HER2+ $\mathrm{BC}$ in patients who have received 2 or more prior antiHER2 regimens. In the phase III SOPHIA study presented at SABCS 2020, among 536 patients with disease pro- gression after at least 2 lines of anti-HER2 therapy, the addition of margetuximab to chemotherapy prolonged PFS relative to trastuzumab plus chemotherapy (median PFS 5.8 vs. 4.9 months), but with similar OS. A survival benefit of 4 months was restricted to patients with the CD16A receptor. Serious adverse events were comparable in the 2 arms.

\section{Foukakis: What is the optimal treatment approach to the patient with HER2+ BC and CNS involvement?}

Matikas: Patients with HER2+ BC and CNS involvement might have prolonged survival, so long-term toxicity becomes a significant factor in designing their management. Local and systemic approaches are complementary to each other and patient management should be individualized. Regarding local therapies, ESMO guidelines support surgical removal of solitary metastases if feasible, followed by focal radiotherapy, and strongly recommend stereotactic radiation instead of whole brain radiotherapy in case of unresectable CNS involvement. Systemic therapy should not be changed in cases of newly developed CNS metastases and stable extracranial disease. Options that have documented efficacy at this setting include trastuzumab emtansine (KAMILLA), trastuzumab deruxtecan (DESTINY Breast-01), and capecitabine/trastuzumab/tucatinib (HER2CLIMB). The place of neratinib and lapatinib following the preferred options is currently uncertain. Finally, leptomeningeal carcinomatosis carries a dismal prognosis; case reports of intrathecal administration of trastuzumab occasionally report long-term control, although no conclusions regarding its efficacy can be drawn.

Oikonomidou: Following from previous question for those patients with stable brain metastases I would offer T-Dxd (PFS was 18 months in this group in Destiny-01), and those with active brain metastases I would offer Tucatinib unless the patient is keen on oral therapy rather than i.v. one. For patients on Tucatinib with intracranial progressing disease but stable extracranial disease I would carry on with Tucatinib despite the intracranial progression and this is based on the interesting data from the substudy analysis beyond first CNS progression in patients who were maintained on tucatinib/capecitabine/ trastuzumab; there was a very nice difference at 7.6 versus 3.1 months favoring the continuation of tucatinib post first progression.

Andreopoulou: Brain metastases pose a frequent clinical challenge for patients with advanced HER2 $+\mathrm{BC}$ with systemic treatment options limited in efficacy than in the distant control of the disease. Biology complexities have 
been addressed with the development of new drugs that cross the blood-brain barrier, as well as clinical trial designs that include patients with active CNS metastases. Data now provide options including small molecule tyrosine kinase inhibitors and ADCs. Lapatinib in combination with capecitabine supported meaningful durable CNS response rates ranging in pretreated patients with higher benefit in the newly diagnosed setting. These results supported the concept of evaluating further HER2targeted tyrosine kinase inhibitors for the treatment of brain metastases. Neratinib as a single therapy or in combination with capecitabine has demonstrated activity in brain metastases. Tucatinib is the newest treatment strategy for active brain metastases in HER2+ metastatic BC. The HER2CLIMB is the first randomized study to demonstrate clinically meaningful improvement in patients with active or treated CNS disease. The regimen tucatinib, capecitabine, and trastuzumab was approved in April 2020 for adult patients with advanced unresectable or metastatic HER2+BC, including patients with brain metastases, who have received one or more prior antiHER2-based regimens in the metastatic setting. Although CNS penetration seems to be limited with antibody-based agents, such as trastuzumab, pertuzumab, or TDM-1 (trasutuzumab emtansine) studies utilizing $89 \mathrm{Zr}$-labeled trastuzumab tracer have suggested there is some penetration through a disrupted blood-tumor barrier. Recent data on T-DM1 have shown activity in brain metastasis. Although data on DS8201 (trastuzumab deruxtecan) in patients with active brain metastases are still lacking most recently a subgroup analysis of patients with CNS metastases at baseline in DESTINY-Breast01 reported patients who had stable or treated brain metastasis, and outcomes were similar to those of patients without CNS disease.

Mavroudis: CNS involvement is common in patients with HER2+ BC. Trastuzumab-emtansine (T-DM1) could be a therapeutic option for patients with HER $2+$ BC and CNS involvement. Multiple case series have reported CNS response rates $20-44 \%$ in HER2+ BC.

The recently approved tucatinib in combination with capecitabine and trastuzumab is another excellent option based on the results of the HER2CLIMB trial. This trial was unique because it allowed enrollment of patients with active brain metastases. Patients with leptomeningeal disease were not included. As previously mentioned, 291 patients had brain metastases at study entry, 174 of whom had active brain metastases (including both untreated and treated, progressing brain metastases). Among all patients with brain metastases, the tucatinib arm improved median OS (18 vs. 12 months) and PFS for CNS (9.9 vs. 4.2 months). Among 75 patients with active brain metastases, the tucatinib group had improved me- dian OS (21 vs. 12 months) and PFS for CNS (9.5 vs. 4.1 months). The intracranial response rate for tucatinib arm was 47 versus $20 \%$ for placebo arm. These results support the use of this regimen rather than whole brain radiotherapy in select patients with brain metastases from HER2 + BC.

\section{Conflict of Interest Statement}

T.F., A.M., O.O., E.A., and D.M.: no conflict of interest to declare.

\section{Chair}

Assoc. Prof. Theodoros Foukakis

Department of Oncology-Pathology, Karolinska Institutet

Breast Center, Karolinska University Hospital

SE-17176 Stockholm, Karolinska Solna (Sweden)

Theodoros.foukakis@ki.se

\section{Participants}

Assoc. Prof. Alexios Matikas

Breast Center, Theme Cancer

Karolinska University Hospital

Gävlegatan 55

SE-17164 Stockholm (Sweden)

Alexios.matikas@ki.se

Prof. Olga Oikonomidou

Cancer Research UK Edinburgh Centre, Institute of Genetics and Cancer

Edinburgh Cancer Centre, Western General Hospital

University of Edinburgh

Crewe Road South

Edinburgh EH4 2XR (UK)

Olga.Oikonomidou@ed.ac.uk

Prof. Eleni Andreopoulou

Breast Cancer Center Clinical Research

Weill Cornell Medicine/New York Presbyterian Hospital

420 East 70th Street, 2nd Floor

New York, NY 10065 (USA)

ela9082@med.cornell.edu

Prof. Dimitris Mavroudis

Department of Medical Oncology

University Hospital of Heraklion

Voutes

GR-71110 Heraklion (Greece)

mavroudis@uoc.gr 


\section{References}

1 Prat A, Guarneri V, Paré L, Griguolo G, Pascual T, Dieci MV, et al. A multivariable prognostic score to guide systemic therapy in early-stage HER2-positive breast cancer: a retrospective study with an external evaluation. Lancet Oncol. 2020 Nov;21(11):1455-64.

2 Loibl S, Majewski I, Guarneri V, Nekljudova V, Holmes E, Bria E, et al. PIK3CA mutations are associated with reduced pathological complete response rates in primary HER2positive breast cancer: pooled analysis of 967 patients from five prospective trials investigating lapatinib and trastuzumab. Ann Oncol. 2016 Aug;27(8):1519-25.

3 Hyman DM, Piha-Paul SA, Won H, Rodon J, Saura C, Shapiro GI, et al. HER kinase inhibi- tion in patients with HER2- and HER3-mutant cancers. Nature.2018 Feb;554(7691):18994.

4 Slamon D, Eiermann W, Robert N, Pienkowski T, Martin M, Press M, et al.; Breast Cancer International Research Group. Adjuvant trastuzumab in HER2-positive breast cancer. N Engl J Med. 2011 Oct;365(14):127383.

5 Tolaney SM, Guo H, Pernas S, Barry WT, Dillon DA, Ritterhouse L, et al. Seven-Year Follow-Up Analysis of Adjuvant Paclitaxel and Trastuzumab Trial for Node-Negative, Human Epidermal Growth Factor Receptor 2-Positive Breast Cancer. J Clin Oncol. 2019 Aug;37(22):1868-75.
6 Schneeweiss A, Chia S, Hickish T, Harvey V Eniu A, Hegg R, et al. Pertuzumab plus trastuzumab in combination with standard neoadjuvant anthracycline-containing and anthracycline-free chemotherapy regimens in patients with HER2-positive early breast cancer: a randomized phase II cardiac safety study (TRYPHAENA). Ann Oncol. 2013 Sep;24(9):227884.

7 Gianni L, Pienkowski T, Im YH, Roman L, Tseng LM, Liu MC, et al. Efficacy and safety of neoadjuvant pertuzumab and trastuzumab in women with locally advanced, inflammatory, or early HER2-positive breast cancer (NeoSphere): a randomised multicentre, open-label, phase 2 trial. Lancet Oncol. 2012 Jan;13(1):25-32. 